Cipango Cahiers d'études japonaises

$20 \mid 2013$

Nouveaux regards sur les arts de la scène japonais I

\title{
Une vie d'itinérance, ensemble jour et nuit
}

Le taishū engeki ou les joies et les peines des troupes théâtrales

familiales dans le Japon d'aujourd'hui

\section{Tomoko Takei}

Traducteur : Pascal Griolet

\section{Q OpenEdition \\ Journals}

Édition électronique

URL : https://journals.openedition.org/cipango/1973

DOI : 10.4000/cipango. 1973

ISSN : 2260-7706

\section{Éditeur}

INALCO

Édition imprimée

Date de publication : 30 octobre 2013

ISSN : 1164-5857

Référence électronique

Tomoko Takei, « Une vie d'itinérance, ensemble jour et nuit », Cipango [En ligne], 20 | 2013, mis en ligne le 17 avril 2015, consulté le 30 juin 2021. URL : http://journals.openedition.org/cipango/1973 ; DOI : https://doi.org/10.4000/cipango.1973

Ce document a été généré automatiquement le 30 juin 2021.

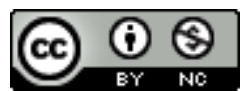

Cipango est mis à disposition selon les termes de la Licence Creative Commons Attribution - Pas d'Utilisation Commerciale 4.0 International. 


\section{Une vie d'itinérance, ensemble jour et nuit}

Le taishū engeki ou les joies et les peines des troupes théâtrales familiales dans le Japon d'aujourd'hui

\section{Tomoko Takei}

Traduction : Pascal Griolet

\section{NOTE DE L'AUTEUR}

Takei Tomoko est une journaliste indépendante. Son reportage a été publié dans le numéro de la revue Fujin kōron daté du 7 mars 2007. Les photographies sont de Tsunoda Takeshi.

\section{Un nouvel acteur est un nouveau-né}

1 Le nombre des troupes itinérantes en tournée à travers le Japon dépasse aujourd'hui largement la centaine. La plupart d'entre elles sont constituées d'un chef, de sa famille et de ses proches auxquels s'agrègent des membres sans lien familial avec eux. Changeant tous les mois de théâtre, ces acteurs mangent et dorment ensemble au fil d'une vie qui est un voyage sans fin.

2 Dix heures du matin. Dans la grande loge commune de la troupe Wakaba Shigeru, Sakura Nagisa, une jeune fille de seize ans, se frotte encore les yeux : elle vient de se réveiller. Elle dépose devant le miroir de chaque acteur une soucoupe remplie d'eau pour diluer la poudre blanche, oshiroi, qui sert à se farder. Nagisa a arrêté l'an dernier le lycée pour faire ses premiers pas d'actrice.

3 Les membres de la troupe arrivent les uns après les autres de leur résidence qui se trouve à deux pas du théâtre. Ils s'annoncent à chaque fois d'une voix sonore. Ai Nozomi (vingt-huit ans) porte sur son dos Aki, sa fille, qui vient d'avoir six mois. Ai Haruka (vingt-quatre ans) est entrée dans la troupe il y a trois ans. Elles s'assurent 
toutes deux du titre de la pièce jouée aujourd'hui et des morceaux de musique sur lesquels seront exécutées les danses. Elles préparent ensuite les costumes et les accessoires qui seront nécessaires. Ai Suzume (trente-huit ans), l'actrice la plus ancienne de la troupe, a commencé dans le coin-cuisine les préparatifs du déjeuner avec les courses qu'elle a faites hier soir après la représentation.

La troupe Wakaba gekidan devant le théâtre Taishōkan, à Asakusa

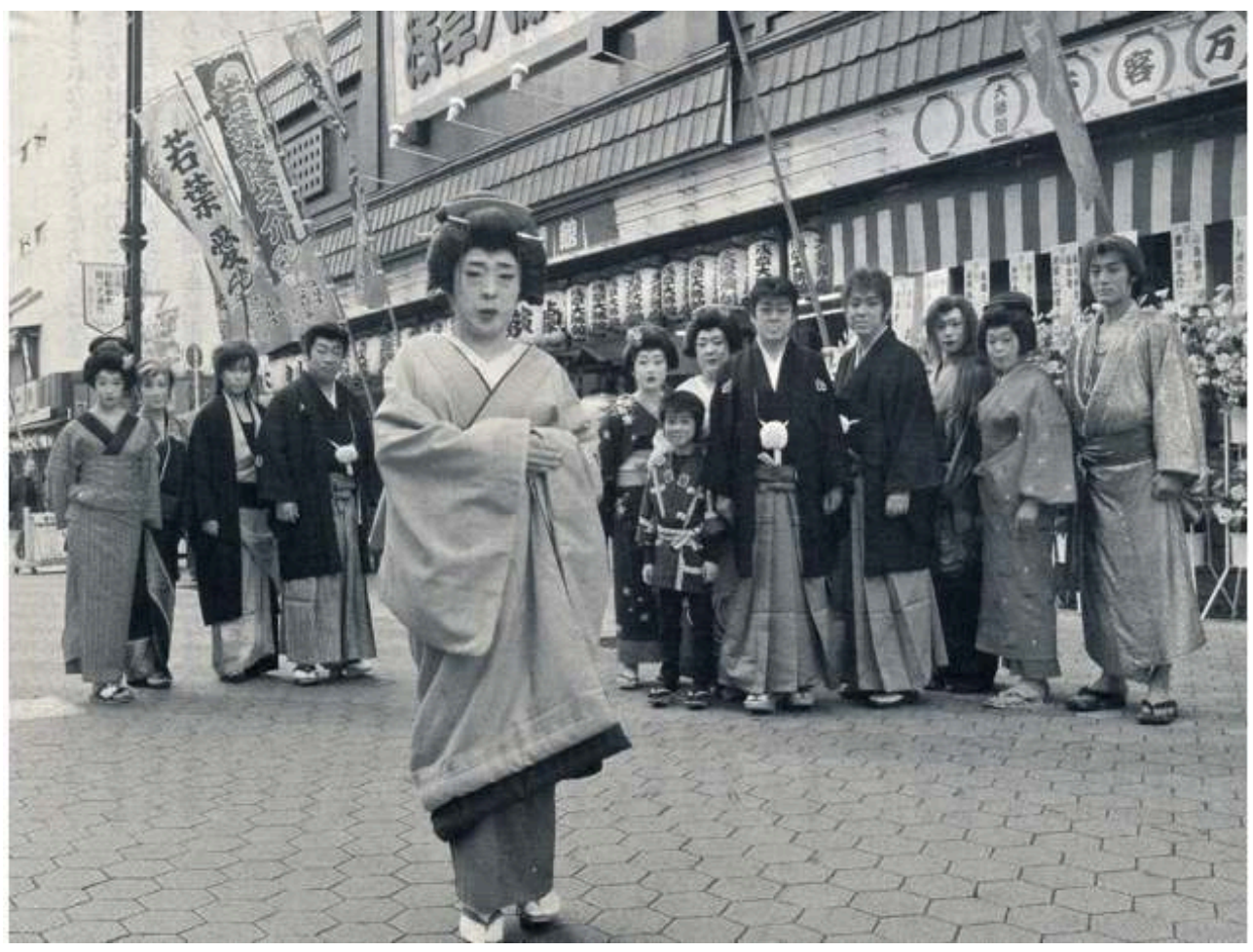

Au centre de la photo, Wakaba Shigeru, fondateur de la troupe, joue depuis un demi-siècle des rôles d'onnagata (figures féminines incarnées par des hommes). Derrière lui, de droite à gauche, Ren Benta, Ai Haruka, Kūma Tairin, Ai Yônosuke en tenue de cérémonie tout comme Wakaba Ai, le fils de Wakaba Shigeru. Ces deux derniers ont le titre de chef de troupe (zachō) et en sont les têtes d'affiche. Viennent ensuite Ai Suzume, la sœur ainée d'Ainosuke, puis Kūma Tenka et Ai Nozomi. À gauche de Wakaba Shigeru, Wakaba Ryūnosuke est en tenue de cérémonie, Umezawa Kikunosuke, hôte de passage, est une actrice qui joue de préférence des rôles d'hommes. Izumi Kaoru est la mère de Tairin et de Tenka. Enfin vient la petite dernière, Sakura Nagisa, qui n'a que seize ans. La troupe compte actuellement onze acteurs. Par le passé, elle en comptait parfois le double. La constitution des troupes fluctue avec le temps

4 Le jeune Ren Benta (vingt-neuf ans) entre en tapant un message sur son portable. «Quand on a du succès auprès des filles, on est très pris dès le matin, pas vrai ? " lui lance Izumi Kaoru (quarante et un ans) qui est en train d'étaler le fard blanc sur le visage de son fils Kūma Tenka (huit ans) devant leur miroir. À côté, Kūma Tairin (quinze ans) se déshabille et enfile un vêtement de dessous en soie blanche. Cette mère a intégré la troupe, avec ses deux enfants, l'an dernier, après avoir divorcé et quitté celle à laquelle elle appartenait.

5 «Tairin, tu es bien mince! J'espère que tu prends un bon petit-déjeuner le matin. Si tu veux, je te donnerai ma part de viande!» La plaisanterie aux lèvres, arrivent deux acteurs confirmés, Wakaba Ryūnosuke (trente-six ans) et Ai Yōnosuke (trente-quatre ans). Ryūnosuke est l'époux de Nozomi. Yōnosuke est le frère cadet de Suzume. Sur la scène, l'éclairagiste Kawaguchi Mitsuo (quarante-trois ans), qui durant tout le spectacle 
manipulera au fond de la salle deux projecteurs de poursuite, vérifie en silence le bon fonctionnement de la régie son ainsi que les décors de la pièce jouée aujourd'hui.

En coulisse

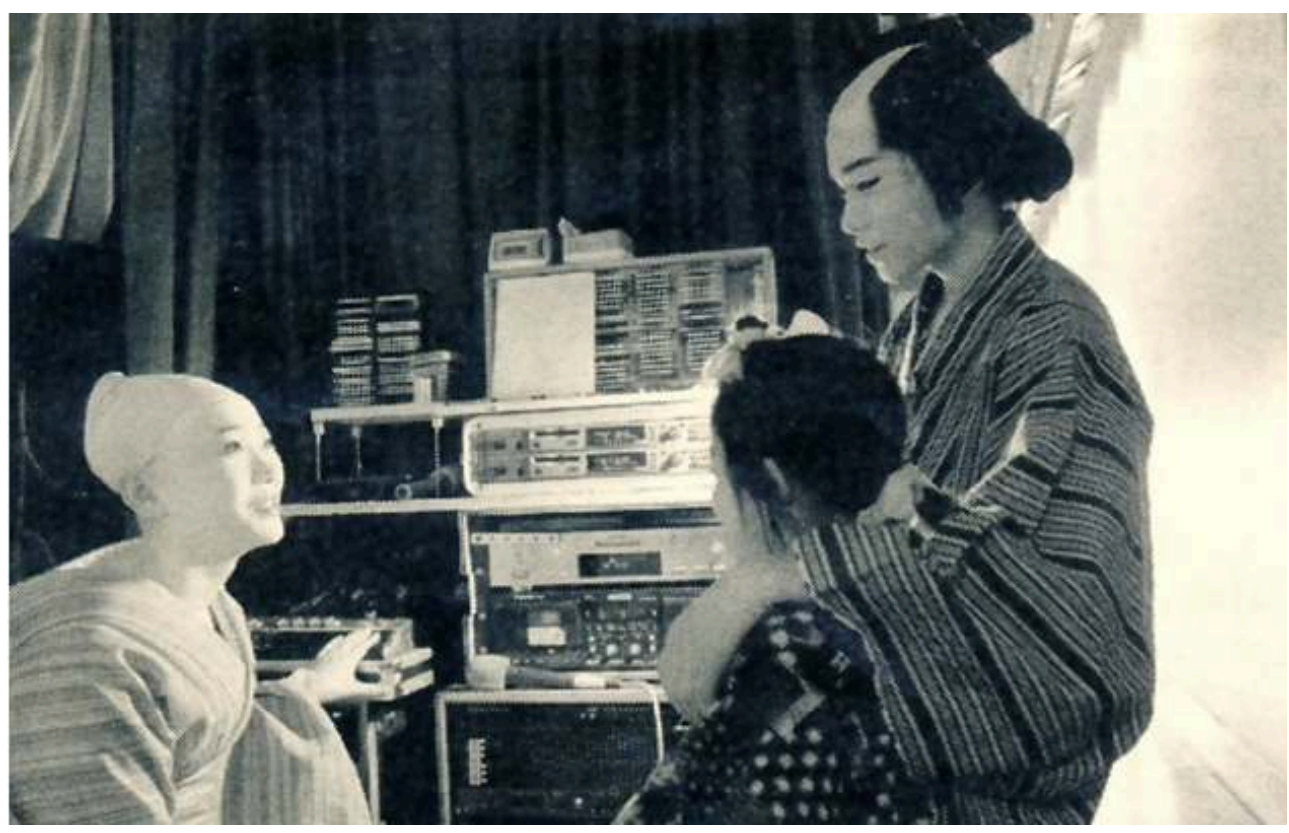

Pendant le spectacle, Nozomi, qui s'occupe de la sonorisation dans les coulisses côté cour, plaisante avec les deux frères. Ceux-ci n'ont pas le même père, mais Tairin, l'aîné, prend soin de son petit frère, Tenka

6 Alors que les acteurs ont à peu près terminé de se maquiller entrent Wakaba Shigeru (soixante-sept ans) et son fils, Wakaba Ai (cinquante ans). Wakaba Shigeru qui est à la tête de la troupe Wakaba gekidan est actif depuis plus de cinquante ans et exerce principalement dans la région du Kantō, autour de Tōkyō. De petite taille et tout en délicatesse dans ses pas de danse, il est l'un des plus célèbres onnagata (acteur jouant des rôles féminins) de ce type de théâtre; il interprète volontiers des femmes du peuple, coquettes et généreuses. Les pièces qu'il a composées sur des histoires très sentimentales sont devenues des classiques.

7 Le taishū engeki connut un succès sans précédent dans les années 1970 et 1980 . Wakaba Shigeru fut presque aussi célèbre qu'Umezawa Tomio, la vedette de la troupe Umezawa Takeo gekidan, la coqueluche de l'époque, que l'on appela le «Tamasaburō des quartiers populaires » (Tamasaburō étant aujourd'hui le plus séduisant et le plus célèbre onnagata du kabuki). En 1994, un documentaire télévisé sur ses petits-fils, Chibitama sankyōdai, "les trois Tama(saburō) en herbe ", rencontra un tel succès qu'on en fit une série durant six ans et que ces enfants de la scène devinrent célèbres à travers tout le Japon. Lorsque le fils de Wakaba Shigeru divorça, ses cinq petits-fils prirent leur indépendance en fondant «la Nouvelle troupe de Wakaba " (Shinsei Wakaba gekidan). Shigeru se retira en 2004 et, depuis lors, ce sont les deux têtes d'affiche, son fils, Ai, et Yōnosuke, un «disciple de la deuxième génération », qui dirigent cette troupe de douze membres, dont onze acteurs.

8 «Considère les membres de la troupe comme ta propre famille!» Tel est le conseil que le père de Shigeru, lui-même acteur, lui donna lorsqu'il se trouva propulsé à la tête d'une troupe à l'âge de seize ans. 
« Depuis toujours, il est d'usage dans notre milieu d'appeler niisan, "grand frère", même son propre père. Ici, les liens de sang ne comptent pas. Les membres d'une troupe sont tous les enfants d'une même famille. Ils sont comme frères et sœurs. Lorsqu'un nouveau membre arrive, je le considère comme un nouveau-né. Ma femme est morte, il y a cinq ans. Outre mon fils, Ai, j'ai aussi une fille qui vit, elle, comme tout le monde de façon sédentaire, mais en fin de compte il n'y a que sur les membres de la troupe que je peux compter.»

Shigeru et Ai sont assis devant les miroirs du fond de la loge. Ils ont commencé à se maquiller d'une main experte. Avant que le spectacle ne commence, les membres de la troupe déjà prêts viennent saluer à tour de rôle en s'agenouillant les mains sur le sol et en inclinant la tête pour dire la formule consacrée Yoroshiku onegai shimasu (« Merci par avance de votre bienveillante indulgence à mon égard»). Shigeru se retourne à chaque fois vers eux pour les saluer à son tour. Il est, dit-on, impoli de saluer à travers le miroir.

Dans la loge

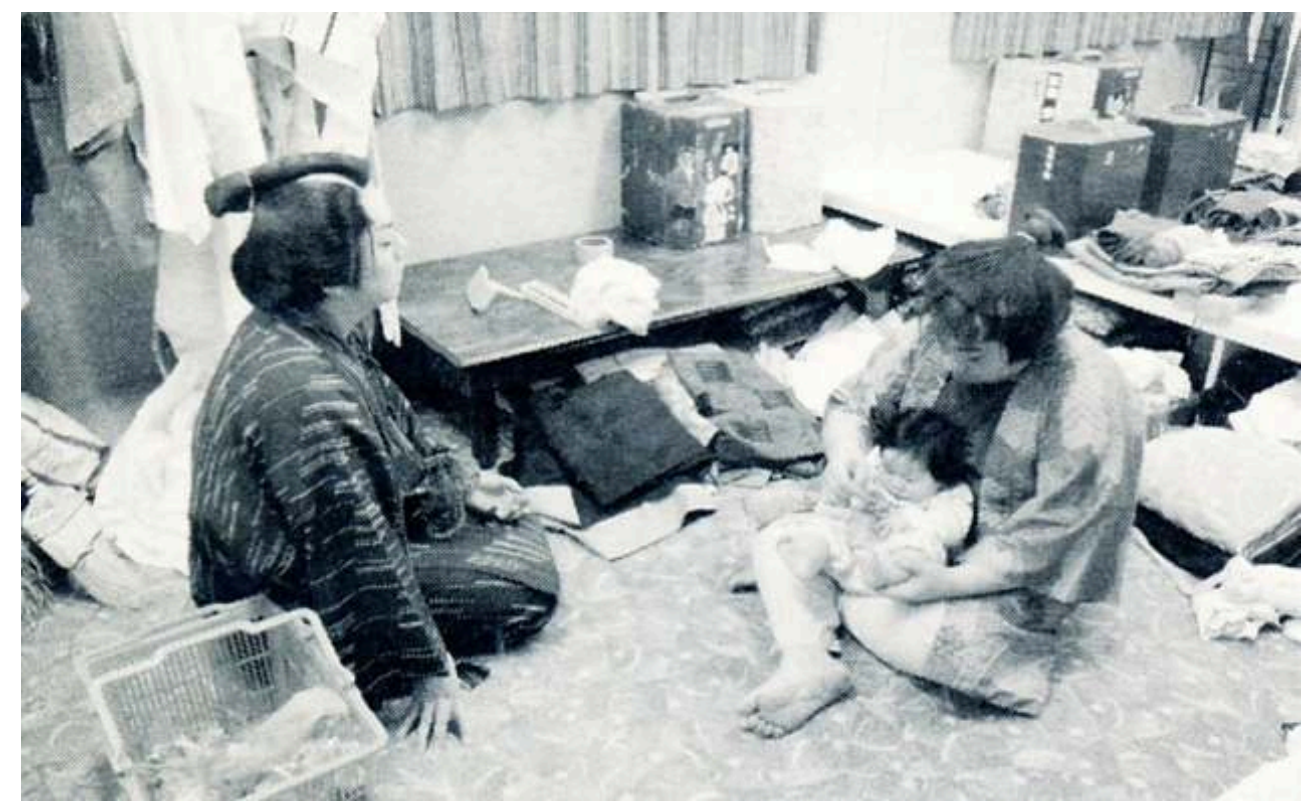

Dans la loge commune, le mari de Nozomi, Ryūnosuke, s'occupe de leur fille, Aki. À ses côtés, Yōnosuke est prêt à entrer en scène. Les membres de la troupe sont très attentifs les uns vis-à-vis des autres et c'est ce qui crée l'harmonie que dégagent leurs relations

11 «Ici, tout commence par les salutations. Peu importe que l'on soit supérieur ou inférieur ! On doit saluer d'une voix énergique. »

Dans le monde du taishū engeki, les usages anciens sont toujours de rigueur et l'on est très à cheval sur l'étiquette et les bonnes manières. Les acteurs construisent des relations de maitre à disciple : l'ordre hiérarchique, tout comme la répartition des rôles entre hommes et femmes, est clair et net. Les travaux de force sont pour les hommes. Les travaux ménagers sont pour les femmes. Le chef de la troupe lui-même ne se permet pas de faire des remarques sur les tâches des femmes dès lors qu'elles en ont la charge. Du chef à l'éclairagiste, tout le monde partage le même repas. 


\section{Les nouveau-nés font partie de la troupe}

13 Dix minutes avant que ne débute la séance de midi, la salle s'est peu à peu remplie. Le spectacle durera trois heures et demie avec, pour commencer, une courte présentation de danses, puis la pièce de théâtre, enfin une succession de danses.

Dès que le rideau s'ouvre, la loge est aussitôt saisie d'une agitation fiévreuse. Lorsqu'ils ne sont pas sur scène, les acteurs assurent toutes les tâches invisibles du public. Ils font fonctionner à tour de rôle la sono, les éclairages ou les projections de fumées depuis les coulisses latérales. Les femmes aident les acteurs à se changer et plient avec adresse les kimono utilisés avant de les ranger. Dans ce monde, on ne saurait être un acteur à part entière si l'on ne maîtrise pas tous les savoir-faire des coulisses. Entre deux actes, les acteurs déplacent et installent les décors. En cas de défaut d'installation, le chef de troupe n'hésitera pas à exprimer son mécontentement, sur scène, pendant la pièce.

Les acteurs chevronnés improvisent des dialogues qui font éclater de rire le public. Quand, en revanche, un débutant comme Tairin trébuche sur un mot, Shigeru, qui tient alors le rôle d'une servante des temps anciens, lui lance pour le stimuler: "Un peu vasouillard, Monsieur le fonctionnaire!» et de la salle fusent les encouragements adressés à Tairin.

Dans les coulisses côté jardin, où se trouve la sono, Haruka se glisse aux côtés de Suzume avant qu'elle ne manipule les commandes. Les effets sonores, comme le claquement des claquoirs de bois, doivent être parfaitement en phase avec la respiration des acteurs. Sur les planches comme dans les coulisses, l'apprentissage du métier se fait en regardant les autres. Tenka, accouru en petite tenue, ne perd rien du jeu des acteurs en scène et lorsque vient son tour, ce sont les anciens qui observent son interprétation. Dans un coin de la loge enfiévrée, Aki se réveille à peine; quelqu'un la prend dans les bras, la berce, change ses langes.

17 Dès que le rideau s'est fermé sur le tableau final auquel ils participent tous, les acteurs s'élancent vers la sortie pour saluer au-dehors les spectateurs et les remercier d'être venus. Les femmes retournent ensuite les premières dans la loge pour y installer une table et dresser le couvert.

18 Lorsque le déjeuner est terminé, il est déjà seize heures. La séance du soir commence à dix-huit heures. Le répit est de courte durée et la loge redevient vite un lieu de travail : l'eau qui sert à diluer la poudre a été renouvelée. Le spectacle de la soirée s'achève vers vingt et une heures trente. Après le dîner, vers vingt-deux heures, tous les membres s'assoient en cercle sur la scène afin de préparer le spectacle du lendemain.

Le programme change tous les jours durant un mois. Les répétitions n'ont d'autre support que la parole : elles reposent sur la transmission orale (appelée kuchidate) du chef de la troupe, sans aucun livret de référence. Wakaba Shigeru raconte à toute allure ce que dit chaque personnage en donnant ses indications sur les combats de sabre ou les changements de scènes. Les membres de la troupe l'écoutent, très attentifs, prenant des notes ou enregistrant ses propos. Les acteurs chevronnés, qui connaissent les dialogues de plus d'une centaine de pièces, se retirent les premiers. Ils expliquent avec application aux plus jeunes les sentiments d'un personnage ou la situation. Les débutants s'exercent aux pas de danse puis mémorisent les dialogues avant de se coucher. Les femmes font le ménage et la lessive, et vers minuit enfin les travaux d'une journée touchent à leur terme. 
Préparation du spectacle du lendemain sur la scène

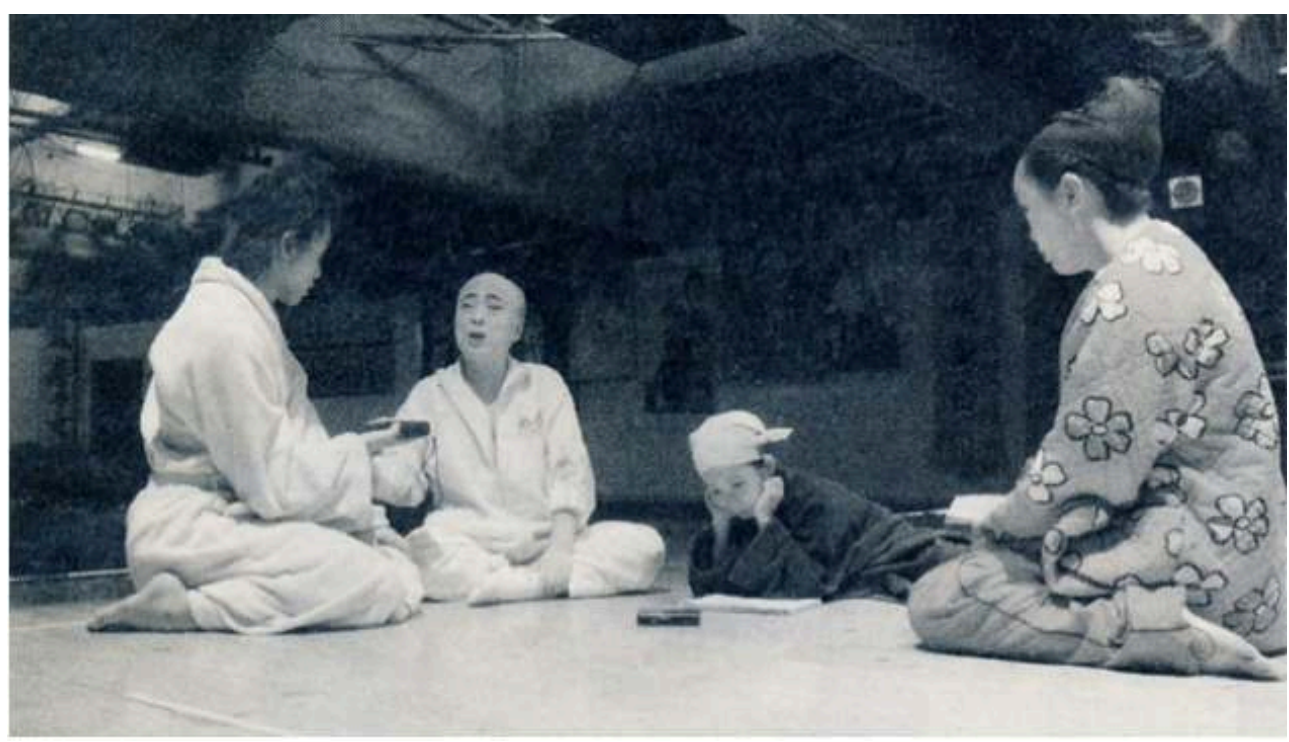

Le soir vers 10 heures, alors que les membres de la troupe ont déjà pour la plupart retiré leur maquillage et pris un bain, la répétition du spectacle du lendemain se fait oralement, dans la tradition du kuchidate. Wakaba Shigeru donne ici ses indications que Tairin enregistre sur son lecteur de cassettes tandis qu'Ai Nozomi prend des notes et que le petit Tenka est pensif

«C'est pénible de changer sans cesse d'école, mais quand on rentre, on retrouve ses parents. Cela vaut mieux que d'éprouver la tristesse des enfants dont les deux parents travaillent. Vers douze ans, j'ai commencé à avoir envie de jouer et à apprendre le métier, mais mon père, aujourd'hui disparu, était très sévère et quand je me trompais, par exemple pour poser l'aiguille sur le sillon d'un disque qui avait été marqué avec du blanc de maquillage, il me frappait avec les claquoirs de bois. Je ne me suis pas révolté, mais je faisais souvent l'école buissonnière lorsque j'étais au collège, au point que ma mère s'en inquiétait. Un jour dans un sauna, un acteur âgé de la troupe me parla de toutes sortes de sujets : il me fit comprendre que c'était une chance d'avoir des parents, qu'il ne fallait pas brimer les plus faibles, qu'il fallait prendre sur soi les responsabilités de ceux que l'on a sous ses ordres. Peu à peu, j'ai évolué... » 


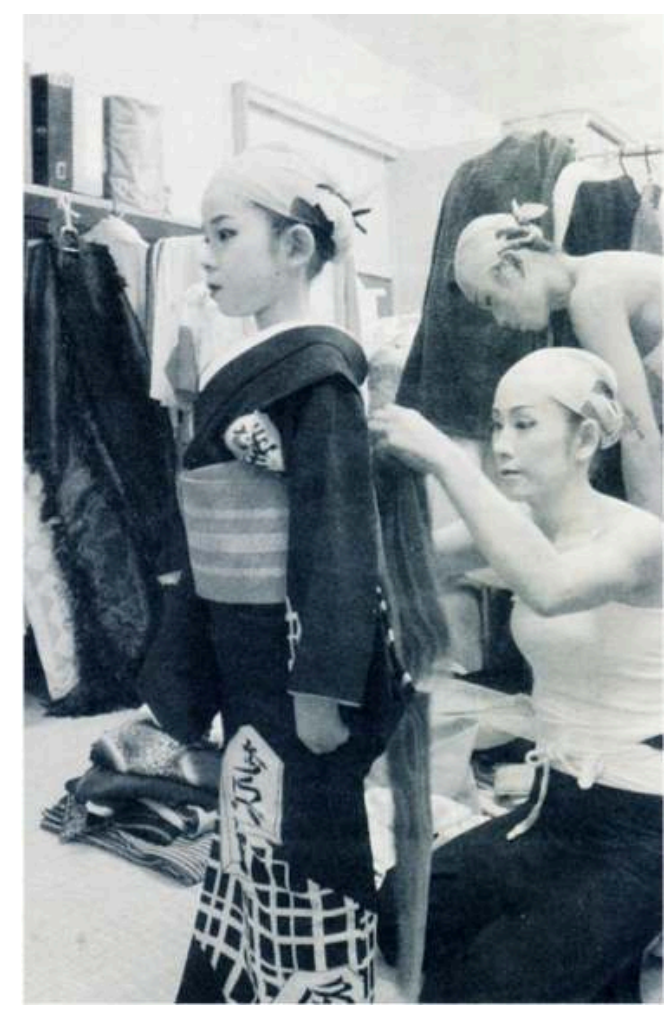

Kaoru doit se préparer elle-même ainsi que ses deux enfants. Tairin qui souffre d'une grave maladie a longtemps été sur une chaise roulante et Tenka fut lui aussi plusieurs fois hospitalisé. Depuis son divorce, elle a joué dans des stations thermales. Sans rien montrer de ses soucis, elle garde le sourire avec vaillance

Suzume, qui était une admiratrice d'Ai, intégra la troupe à l'âge de quinze ans. C'était l'âge d'or du taish ù engeki. « Mon maître (Ai), comme le grand-maître (Shigeru), une fois le travail terminé, disparaissaient aussitôt pour aller voir des admiratrices et, la première année, ils ne m'ont pas adressé une seule fois la parole. Ma première tâche fut de servir le café à la "grande sœur", la mère des enfants-acteurs qui ont pris maintenant leur indépendance. À l'époque, elle régnait en maître sur la vie dans les coulisses. Elle était dure : pendant le spectacle, je devais plier cinq kimono le temps d'une danse. Quand j'y suis parvenu, il m'a fallu les plier le temps d'un couplet et demi, puis d'un seul couplet. C'était de plus en plus difficile. Mais j'ai continué à corps perdu et, au bout de plusieurs années, je suis parvenu à travailler comme les autres et j'en suis venu à bavarder normalement avec ma grande sœur. Elle m'enseigna comment coudre les dessous de kimono. Ce que j'ai ainsi acquis à force d'endurance constitue aujourd'hui mon plus précieux trésor. »

Un acteur auquel les spectateurs rendent hommage par des dons en espèces offerts au cours des spectacles de danse peut enrichir sa garde-robe de kimono somptueux, mais, dans la phase d'apprentissage, le salaire n'est qu'un simple pourboire; ainsi, lorsque Yōnosuke entra dans la troupe à sa sortie du lycée, certains mois, il ne pouvait même pas s'acheter de jus de fruits.

"À cette époque, la grande sœur (qui était l'épouse du chef de la troupe) me donnait de l'argent en me disant: "Ne le dis à personne !" Lorsque j'ai commencé enfin à recevoir des hommages de la part de spectateurs, j'ai voulu la rembourser, mais elle a refusé 
tout net en me suggérant d'acheter quelque chose pour la troupe. Je me suis souvent disputé avec elle au sujet de la façon d'élever les enfants, mais je lui dois beaucoup. »

Les enfants d'une compagnie sont modelés par la grande famille que celle-ci constitue et deviennent eux-mêmes des acteurs.

\section{Les secrets de la respiration commune}

Au lycée, Nozomi faisait partie du club de théâtre ; lorsqu'elle entra dans la troupe, il y avait déjà plusieurs filles de son âge et quand l'une d'entre elles se trompait, la responsabilité retombait sur elles toutes; aussi leurs relations n'étaient-elles pas toujours faciles.

"Mais, on était tous les jours ensemble. On ne pouvait pas rester longtemps de mauvaise humeur. On se réconciliait à chaque fois. Depuis, lorsque j'assiste à une dispute, je ne m'en mêle pas. Avec le temps, les choses se remettent en ordre. Lorsqu'on me disait qu'il fallait être toujours ensemble, que ce soit pour aller faire des courses ou aux toilettes, j'avais du mal à l'admettre, mais aujourd'hui je me dis que cela signifiait qu'il ne fallait pas s'isoler dans son coin, mais toujours coopérer. »

Ne pas se mêler de la vie privée des autres semble être une règle tacite. Mais il y a peu de temps libre. Aussi, il se limite bien souvent à se détendre avec des vidéocassettes, des jeux électroniques ou en faisant des courses. Là où ils oublient tous les ennuis du monde, c'est sur scène. Le plus grand encouragement, c'est le sourire des spectateurs. C'est ce que tous répètent.

28 La femme d'un acteur connaît ses admiratrices, mais elle ne dit rien quand il sort après le spectacle avec elles. Elle ne dit rien non plus s'il va s'amuser avec des filles de joie. En revanche, les relations amoureuses sont interdites au sein de la troupe : c'est pourquoi Ryūnosuke et Nozomi ont dû attendre plus d'un an pour se fréquenter.

Les acteurs ont au maximum un jour de congé par mois, mais ils ne font jamais relâche, sauf en cas de maladie ou d'accident. À l'âge de vingt ans, Ai a eu maille à partir avec des yakuza. L'un d'eux l'a menacé jusque devant la loge avant le spectacle: «Si tu montes comme ça sur scène, je te tue ». Mais Shigeru, son père, l'aurait alors exhorté : «Obtiens au moins qu'il te laisse jouer maintenant ! ».

«Lorsque je suis revenu le voir, tremblant, la peur au ventre, pour lui demander cette autorisation, le yakuza m'a félicité en me disant que j'avais du cran! Plus qu'un père, le chef de la troupe est un acteur que j'admire. Je ne me suis jamais révolté, même quand il me passait des savons. On a beau se disputer dans la loge, sur scène on s'embrasse en se disant des mots tendres. "

31 Cette vie de voyage ne laisse guère de liberté. Mais en partageant les joies et les peines de la scène et des coulisses se tissent des liens qui permettent de se comprendre à demi-mot et de communiquer sans paroles.

Avant d'avoir vingt ans, Ai ne se voyait guère confier de rôle et décida de s'en aller. Après avoir vécu de petits boulots pendant deux ans, puis avoir été engagé dans la troupe d'une connaissance, il fut rappelé par son père, Shigeru, qui lui dit: «Allez, reviens ! Je vais te donner des rôles. »

33 « Or, à mon retour, je n'étais pas capable de bien jouer et le chef m'a frappé avec le coin du dos d'un bottin téléphonique. Alors, de dépit, je me mis à étudier. » 
Avant d'avoir vingt ans et de monter à Tōkyō depuis la région du Kansai à la tête de sa troupe, Shigeru lui-même avait fui la sienne : attiré par Tōkyō, il était entré dans une troupe féminine qui rencontrait alors un très grand succès dans le quartier d'Asakusa, avec à sa tête l'actrice Fuji Yōko. Quelques mois plus tard, son père est venu le rechercher. Par la suite, lui-même a fait l'expérience douloureuse de voir la moitié des membres de sa troupe s'évaporer. Il a alors ressenti fortement que «réunir de bons acteurs, ce n'est pas disposer d'une belle distribution. Plus que le talent de chacun, ce qui importe c'est le lien qui nous unit ».

Le répertoire

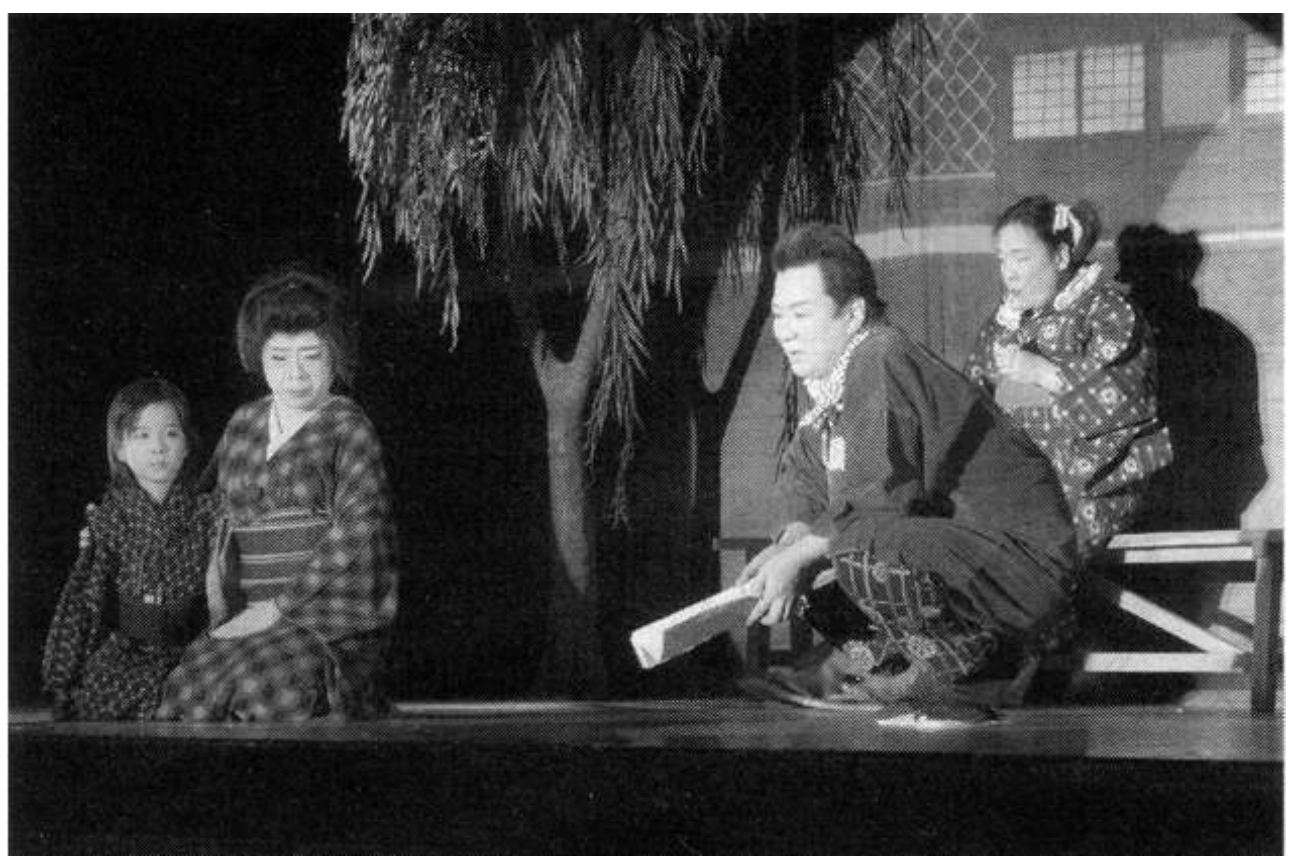

La troupe de Wakaba joue aussi bien des pièces d'avant-guerre comme la Mère au fond des yeux (Mabuta no haha) que d'après-guerre comme la Mère sur la falaise (Ganpeki no haha)

Dans le taishū engeki, les pièces qui traitent des conflits entre devoir et sentiments ou de l'affection entre parents et enfants touchent toujours le cœur d'un large public. Peutêtre est-ce ce lien de fraternité entre les acteurs qui les rend capables d'émouvoir un large public.

"Ceux qui s'en vont ne cessent pas de jouer pour autant. En parcourant le monde, ils découvrent la valeur de la troupe. Il m'arrive même de les pousser à partir pour se former. Même ceux qui ont disparu en douce, je les accueille à leur retour sans leur faire de reproches. C'est cela une famille. »

C'est sans doute en les coupant qu'ils comprennent la valeur des liens qui les unissent les uns aux autres.

\section{Le sentiment profond du bonheur}

Alors que, depuis vingt ans, les familles nucléaires ont considérablement augmenté au Japon, Wakaba Shigeru crée des pièces qui invitent à rire ou à pleurer sur le thème de la maternité. Il s'agit toujours "d'une mère imparfaite»: soit, par coquetterie, elle fait 
passer sa fille pour sa petite sœur; soit, elle est une geisha sur le retour qui est une source permanente d'ennui pour sa fille; soit encore, en raison de son manque d'éducation, elle fait échouer le projet de mariage de sa fille. Elle se résout alors à renoncer à l'alcool ou aux choses de l'amour pour apprendre enfin à lire et à écrire. C'est toujours l'image d'une mère qui, bien que maladroite, souhaite du fond du cœur le bonheur de ses enfants.

"J'ai appris des spectateurs que le charme de la vie se trouve dans des choses sans importance. La profondeur du sentiment d'être heureux change avec l'expérience des épreuves. Dans la vie quotidienne qui nous garantit, croyons-nous, le bonheur, il suffit que le chef de famille se trouve au chômage pour que tout s'écroule : aussitôt, les liens familiaux se distendent. Si l'on partage les peines en famille, un simple petit bonheur prend une profondeur beaucoup plus grande.»

40 Cette « mère imparfaite » incarnée sur scène parvient toujours à saisir un petit bonheur grâce à l'affection et au soutien de la famille et des autres.

41 «Si un acteur ne peut que donner cinquante pour cent de lui-même, tous ensemble on parvient à cent pour cent. C'est cela la scène du taishū engeki. Si l'un des acteurs s'effondre, un autre, sans rien dire, l'emmène à l'hôpital. Nous constituons une famille et nous comptons les uns sur les autres. Si on se voit confier une lourde charge de travail, c'est parce qu'on sait se rendre utile; du reste on trouvera toujours à se faire aider. C'est ainsi que les membres de la troupe se soutiennent mutuellement. Enfin, qu'ils soient bons ou non sur scène, il importe de manifester le plus grand respect pour ceux et celles qui sont âgés. »

\section{La force des liens}

À la fin de l'an dernier, une épidémie de grippe frappe la loge de la troupe Wakaba gekidan, alors à Ashikaga, ville du département de Tochigi. Cinq minutes avant le début de la représentation, Haruka doit s'aliter. Dans l'urgence, Nozomi la remplace. Le jour des adieux, il pleut. Les hommes, qu'ils aient ou non de la fièvre, doivent transporter les affaires jusqu'au camion sous la pluie. Pour les fêtes de fin d'année, chacun rentre dans son pays natal. 


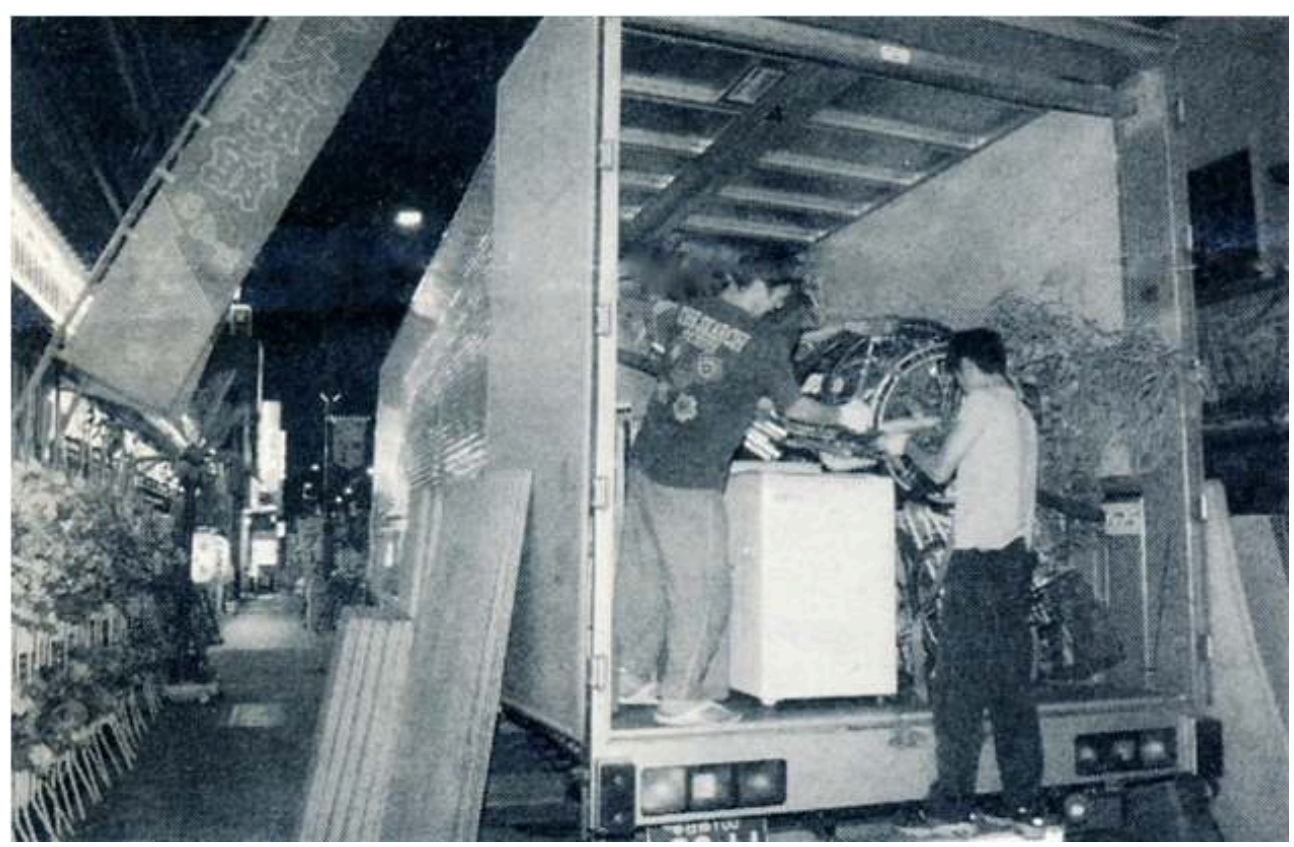

Après la dernière représentation, qui a toujours lieu en matinée, le chargement du ou des camions prend plusieurs heures. Dans la nuit, les acteurs reprennent la route

Dès le jour du Nouvel An, la troupe se reconstitue. Nagisa et Tenka sont plus rayonnants que l'an dernier et saluent avec fermeté. Tairin, qui regrettait un peu de ne pas être allé au lycée, déclare à présent: "Je ne me sens plus perdu. Ma mère me gronde dix fois par jour, mais je suis heureux au sein de la troupe.» Haruka raconte en riant que lors de son retour dans sa famille, elle utilisa des formules honorifiques à tort et à travers sans s'en rendre compte, et s'en trouva ridicule :

44 «Je saluais cérémonieusement ma mère qui venait me secouer le matin pour me réveiller. Mon père, qui s'était opposé à ce que je devienne actrice, aime en fait beaucoup le théâtre. Il raconte à ma mère avec passion les pièces où il m'a vue. Ma "grande sœur", Suzume, depuis qu'elle sait que ma mère a des névralgies, me prévient quand elle voit que la météo est à la pluie dans la région de Chiba. Je pense davantage maintenant à mes parents. »

Leur vie d'« herbes flottantes » sans cesse à la dérive les entraîne d'un endroit à l'autre. Mais qu'il s'agisse de leur famille d'origine ou de celle que constitue leur troupe, ces acteurs transmettent, par leur union renouvelée chaque jour sur scène, l'importance de la chaleur humaine et des liens qui unissent les membres d'une même famille. 